\title{
STABILITY OF ELLIPTICAL GALAXIES. THEORETICAL ASPECTS
}

\author{
V.L. Polyachenko \\ Astrosovet \\ Ul. Pyatnitskaya 48 \\ 109017 Moscow Sh-17 \\ U.S.S.R.
}

\section{INTRODUCTION}

Stars, which contain more than $90 \%$ of the visible matter of the Universe, are organized into systems comprising a hierarchy of different masses and scales: from galactic stellar clusters to clusters of galaxies themselves. Most of the systems in this hierarchic sequence are collisionless: the characteristic time between collisions is much more than typical dynamical times (e.g., the period of oscillations). Various conditions of formation of collisionless gravitating systems leave traces on their main characteristic parameters-such as the angular momentum, the degree of anisotropy, the kind of dependence of the stellar density on coordinates, and so on. The lifetime of a system with a certain set of parameters depends essentially on whether these parameters belong to a region in which the system is stable, or not. If they do, the system may then exist practically without changes for an arbitrarily long time (in terms of the dynamical time scale); if they do not, it must quickly adjust to another, stable state.

Thus, the main question for each special system of whether it is stable or in a state of dynamical relaxation is answered by asking whether the parameters of the system belong to stable or unstable regions. Determination of the boundaries between these two regions is the main problem for stability theory. The importance of the problem is sufficiently evident when studying various collisionless systems, in particular, elliptical galaxies, which have determined in many respects the development of contemporary astrophysics. It is important to note that the requirement of stability imposes actually very essential limits on permissible stationary models of collisionless gravitating systems (it follows from the results below; for details see Fridman \& Polyachenko 1984, hereafter FP).

\section{REVIEW OF EARLIER WORK}

Many of the main results on the stability of collisionless ellipsoidal systems are already published in English and so are easily accessible to Western readers (first of all, see FP). I shall briefly remark on these results and the history of the problem at the beginning of the talk, and devote the main part of the talk to some new results which are not yet available in English translation. 
Studies of the stability of stellar systems already have a sufficiently interesting history of their own. But when we discuss the origins of such work, we should keep in mind the theory of figures of equilibrium and the stability of different geometrical shapes of an incompressible fluid (sphere, ellipsoid, ring, etc.) which was created by many outstanding scientists. The results of their research have been summarized in a large number of reviews and monographs. It is clear, however, that the incompressible fluid approximation (and the corresponding mathematical formalism), generally speaking, is not suitable for describing collisionless systems.

It is quite natural that some concepts and methods used in the equilibrium and stability theory of gaseous gravitating systems proved to be relevant for describing stellar clusters. We should mention here, first of all, the concepts connected with the name of Jeans, who was the first to investigate the stability of a homogeneous distribution of compressible matter: the Jeans instability, the Jeans critical wavelength, etc. The similarity between stellar and gaseous systems was confirmed in the pioneering investigations of collective effects in a collisionless gravitating medium carried out by Antonov (1960), Lynden-Bell (1962), and others. For example, the search for stationary states of stellar systems is equivalent in some cases to solving the corresponding problem in the equilibrium theory of gaseous spheres. Moreover, it turns out that the problem of the stability of such collisionless systems can also be reduced to the stability of certain analogous hydrodynamical systems. However, it should be noted that this "hydrodynamical analogy" (strictly proved by Antonov 1962, and Lynden-Bell \& Sanitt 1969) occurs only for a very special class of isotropic collisionless systems with distribution functions depending on the total energy of a star only. In such a case it is really possible to draw an analogy between the characteristics of the two systems under consideration (for instance, the stellar velocity dispersion then is analogous to the thermal particle velocity of the gaseous system, etc.).

But the uniqueness of collisionless systems consists just in the possibility of anisotropy: the velocity dispersions along different directions may differ strongly from each other. Anisotropy often arises in a natural way-for instance, at the origin of a system; it is typical for various real systems (stellar clusters, galaxies, clusters of galaxies). At the same time, a tradition of applying the hydrodynamical analogy had led to the opinion that stellar clusters are as stable or even more stable than gaseous systems. However, this is correct only for isotropic distributions where the "collisionlessness" reveals itself in the behaviour of perturbations on very small scales only. In real (anisotropic) collisionless systems, not only "old" instabilities (characteristic also of the gaseous medium - for instance, the Jeans instability) are strongly modified, but also new types of instabilities appear which are due to the anisotropy of the velocity distribution of particles, the occurrence of beams, etc.

In the late sixties, after the discovery of quasars, there arose the idea to construct models of spherical stellar clusters with a large redshift. In this connection Mikhailovsky, Fridman and others have carried out a series of investigations on the study of the stability of stellar systems with purely circular orbits (Michailovskii, Fridman \& Epel'baum, 1970; Fridman \& Shukhman 1972; Shukhman 1973; Synakh, Fridman \& Shukhman 1972). Those systems were apparently the first examples of essentially anisotropic systems whose stability was investigated in detail and for which in some cases exact spectra of oscillations were obtained. It follows from these papers that stellar clusters with circular orbits and an outwardly decreasing density are probably stable.

The situation with respect to stability theory of stellar systems in the early 
seventies may be summarized as follows (restricting ourselves to the example of spherical systems). By that time the stability of systems with isotropic distribution functions had been proved and, besides, it had been shown that systems with circular orbits were apparently also stable (excluding a few unphysical ones). Thus there emerged the opinion that all spherical systems should be stable. Such an opinion looked natural, especially if we recall the traditional view that the spherical shape is the most stable one.

However, in 1972 Schukhman and I noticed that systems with nearly radial orbits should be unstable and stated the following problem: to derive the critical anisotropy separating stable and unstable clusters (Polyachenko \& Shukhman, 1972; Polyachenko 1972). That problem was resolved in our subsequent papers, the main results of which were presented in FP. The instability is due to the lack of velocity dispersion in transversal directions and, consequently, has the Jeans nature. If we consider any narrow cone with its center in the center of a sphere and imagine that at the initial time we have slightly compressed it into a new cone by bringing its generatrices together, the inevitability of further Jeans contraction is evident if one takes into account that the particles cannot escape from the perturbation region. A study of the stability of systems with such an anisotropy is important since, on the one hand, it arises naturally at the center of stellar systems, and, on the other hand, stellar orbits in spherical and elliptical galaxies are actually strongly radially elongated.

Due to the importance of these problems I shall return to them below in order to consider the radial instability in a new light and to present more exact stability computations for certain realistic models of spherical stellar clusters, and to outline possible further work in this field.

All the problems mentioned above are concerned with the stability of stationary spherical collisionless systems. But it is no less interesting to ask what role possible instabilities may play in the evolution of systems initially far from equilibrium. For example, one of the most important problems in the theory of galaxy or cluster formation is that of the collapse of a spherical cloud of collisionless particles. In the case of a cloud that is sufficiently cold at the initial moment, practically all the energy released during the contraction converts into radial motion. Consequently, a strong radial orbit instability may develop. As a matter of fact, the conclusion that an ellipsoidal deformation should arise during the collapse of such a cloud was trivial after the "bar-like" instability of corresponding stationary systems were proved; it was made earlier in the author's paper (Polyachenko 1981), dealing, mainly, with instabilities of stationary systems (some more details are presented in Polyachenko 1985). Dr. D. Merritt has recently determined the minimum dispersion of initial transverse velocities in a collisionless cloud that is necessary to ensure its stability (see his paper in this volume).

Then I shall consider the stability of spherical systems with the opposite kind of anisotropy (i.e., with nearly circular orbits). Investigations of such systems were at the beginning mainly of methodological interest. However, the situation has now changed. For instance, it is important to study the processes occurring in the stellar component of dense galactic nuclei, collapsed objects (e.g., black holes), whose vicinities should, evidently, be rich with stars having nearly-circular orbits: stars with elongated orbits having been "absorbed" by a central body. For these systems it was possible to obtain the dispersion relation describing short wavelength perturbations (of the type of the well-known Lin-Shu dispersion relation for disklike galaxies). The physical mechanism of instability which is evident for systems 
with nearly radial orbits (of course, of the Jeans nature) is not yet so evident for the nearly-circular orbits systems. One may give some arguments that this instability has a "beam" nature.

The above listed results were obtained by the same method, using the reduction procedure which will now be proved. This procedure reduces the stability problem for arbitrary spherical collisionless systems to a more simple problem on the stability of the corresponding cylindrical systems relative to flute-like perturbations (when the component of the wave vector along the axis of the cylinder equals zero).

\section{REDUCTION PROCEDURE}

The kinetic equation in the variables $r, \theta, \varphi, v_{r}, v_{\perp}$ and $\alpha$ (where $r, \theta, \varphi$ are the standard spatial spherical coordinates, $v_{r}$ is the radial velocity component, $v_{\perp}^{2}=v_{\theta}^{2}+v_{\varphi}^{2}$ with $v_{\theta}, v_{\varphi}$ are the velocity components in the $\theta$ and $\varphi$ directions, and $\alpha=\arctan \left(v_{\varphi} / v_{\theta}\right)$ ) has the form:

$$
\begin{aligned}
\frac{\partial f}{\partial t} & +\frac{v_{\perp}}{r}\left[\cos \alpha \frac{\partial f}{\partial \theta}+\frac{\sin \alpha}{\sin \theta} \frac{\partial f}{\partial \varphi}-\sin \alpha \cot \theta \frac{\partial f}{\partial \alpha}\right] \\
& +v_{r}\left(\frac{\partial f}{\partial r}-\frac{v_{\perp}}{r} \frac{\partial f}{\partial v_{\perp}}\right)+\left(\frac{v_{\perp}^{2}}{r}-\frac{\partial \Phi}{\partial r}\right) \frac{\partial f}{\partial v_{r}} \\
& -\frac{1}{r}\left(\cos \alpha \frac{\partial \Phi}{\partial \theta}+\frac{\sin \alpha}{\sin \theta} \frac{\partial \Phi}{\partial \varphi}\right) \frac{\partial f}{\partial v_{\perp}}-\frac{1}{r v_{\perp}}\left(\sin \alpha \frac{\partial \Phi}{\partial \theta}-\frac{\cos \alpha}{\sin \theta} \frac{\partial \Phi}{\partial \varphi}\right) \frac{\partial f}{\partial \alpha}=0
\end{aligned}
$$

Consider small perturbations of the sphere with the equilibrium distribution function $f_{0}=f_{0}\left(E, L^{2}\right)$, where $E$ is the stellar energy, $E=\frac{1}{2}\left(v_{r}^{2}+v_{\varphi}^{2}\right)+\Phi_{0}(r)$, with $\Phi_{0}$ the gravitational potential, $L^{2}=r^{2} v_{\perp}^{2}$ is the square of the stellar angular momentum. Such a system is evidently non-rotating. Then the linearized kinetic equation can be presented as

$$
\frac{\partial f_{1}}{\partial t}+\frac{v_{\perp}}{r} \hat{L} f_{1}+\hat{D} f_{1}=\frac{\partial \Phi_{1}}{\partial r} \frac{\partial f_{0}}{\partial E} v_{r}+\frac{1}{r} \hat{L} \Phi_{1}\left(\frac{\partial f_{0}}{\partial E} v_{\perp}+\frac{\partial f_{0}}{\partial L^{2}} 2 v_{\perp} r^{2}\right)
$$

where we introduced the operators:

$$
\begin{gathered}
\hat{L}=\cos \alpha \frac{\partial}{\partial \theta}+\frac{\sin \alpha}{\sin \theta} \frac{\partial}{\partial \varphi}-\sin \alpha \cos \theta \frac{\partial}{\partial \alpha} \\
\hat{D}=v_{r} \frac{\partial}{\partial r}-\frac{v_{r} v_{\perp}}{r} \frac{\partial}{\partial v_{\perp}}+\left(\frac{v_{\perp}^{2}}{r}-\frac{\partial \Phi_{0}}{\partial r}\right) \frac{\partial}{\partial v_{r}}
\end{gathered}
$$

The operator $\hat{L}$ has the standard form of the infinitesimal rotation about the $y-$ axis (being expressed through the Euler angles). ${ }^{1}$ In the case of full spherical

1 Possibly, it was first noted, in the aspect interesting to us, in the unpublished work: M.Ya. Pal'chik, A.Z. Patashinskii, V.K. Pinus and Yu.G. Epel'baum, Institut Yudarnoi iziki Sibir Otd. Akad. Nauk SSSR, 99-100, Novosibirsk, 1970 (In Russian). 
symmetry, the angular part of the potential may be separated in a form proportional to the spherical harmonics: $\Phi_{1} \sim Y_{\ell}^{m}(\theta, \varphi)$. The following calculations may also be considered as the formal justification of this natural statement.

It is natural to solve the kinetic equation in a system of coordinates in which the operator $\hat{L}$ is diagonal, i.e., corresponds to a rotation around the $z^{\prime}$-axis of the turned coordinate system. In this system we present the perturbation of the distribution function in the form of the expansion:

$$
f_{1}=\sum_{s} f_{s}\left(r, v_{r}, v_{\perp}\right) T_{m s}^{\ell}\left(\varphi^{\prime}, \theta^{\prime}, \alpha^{\prime}\right)
$$

where the functions

$$
T_{m s}^{\ell}\left(\varphi_{1}, \theta, \varphi_{2}\right)=e^{-i m \varphi_{1}-i s \varphi_{2}} P_{m s}^{\ell}(\cos \theta),
$$

are introduced, and the $P_{m s}^{\ell}(\cos \theta)$ are the three-index functions (Vilenkin, 1968; see also FP), in particular the $P_{m 0}^{\ell}(\cos \theta)$ functions, identical (except for constant coefficients) to the associated Legendre functions. Therefore it is convenient to write the potential in the form

$$
\Phi_{1}=\chi(r, t) T_{m 0}^{\ell}(\varphi, \theta, \alpha),
$$

or, in the "primed" system,

$$
\Phi_{1}=\chi(r, t) \sum a_{s}^{\ell} T_{m s}^{\ell}\left(\varphi^{\prime}, \theta^{\prime}, \alpha^{\prime}\right),
$$

where the $a_{s}^{\ell}$ are the coefficients of the rotation transforming the $y$-axis into the $z$-axis. Thus, in the "primed" system we have an independent equation for each function of the expansion (4). Taking into account the relation $\hat{L} f_{s}=i s f_{s}$, these equations can be written as

$$
\frac{\partial f_{s}}{\partial t}+\frac{v_{\perp}}{r} i s f_{s}+\hat{D} f_{s}=\frac{\partial \Phi_{s}}{\partial r} \frac{\partial f_{0}}{\partial v_{r}}+\frac{i s \Phi_{s}}{r} \frac{\partial f_{0}}{\partial v_{\varphi}} .
$$

Equation (8) can be presented in a form identical to the standard equation for the response of a cylindrical (or disk) system $f_{s}$ to the flute-like perturbation of the potential, $\Phi_{s}(r, \varphi)=\Phi_{s}(r) e^{i s \varphi}$ (where $r$ and $\varphi$ are the corresponding cylindrical coordinates); if we denote for uniformity $v_{\varphi} \equiv v_{\perp}$, then

$$
\begin{gathered}
\frac{\partial f_{s}}{\partial t}+v_{r} \frac{\partial f_{s}}{\partial r}+\frac{v_{\varphi}}{r} \frac{\partial f_{s}}{\partial \varphi}+\left(\frac{v_{\varphi}^{2}}{r}-\frac{\partial \Phi_{0}}{\partial r}\right) \frac{\partial f_{s}}{\partial v_{r}}-\frac{v_{r} v_{\varphi}}{r} \frac{\partial f_{s}}{\partial v_{\varphi}}= \\
\frac{\partial \Phi_{s}}{\partial r} \frac{\partial f_{0}}{\partial v_{r}}+\frac{1}{r} \frac{\partial \Phi_{s}}{\partial \varphi} \frac{\partial f_{0}}{\partial v_{\varphi}} .
\end{gathered}
$$

Thus, for solving the initial "spherical" problem, it is sufficient to find solutions of the corresponding "cylindrical" problem (9) for all integers $s$ with $-\ell \leq s \leq \ell$ that are even or odd (see below). 
All the above forms the first part of the reduction procedure considered in this section. The second part of this procedure consists in the recipe for calculating the full density perturbation $\rho_{1}$.

Let us suppose that equations (9) for $f_{s}$ are solved. Then for the calculation of $\rho_{1}$, it is convenient to turn in the expansion (4) again to the initial ("non-primed") system, which may be done by means of the formula

$$
T_{m s}^{\ell}\left(\varphi^{\prime}, \theta^{\prime}, \alpha^{\prime}\right)=\sum_{s^{\prime}} T_{m s^{\prime}}^{\ell}(\varphi, \theta, \alpha) \bar{a}_{s^{\prime}}^{\ell} .
$$

Since the expression for the density perturbation $\rho_{1}=\int f_{1} v_{\perp} d v_{\perp} d v_{r} d \alpha$ includes the integration over the angular variable $\alpha$, only one term (corresponding to $s^{\prime}=0$ ) remains from each sum (10), and we obtain the desired formula in the form

$$
\rho_{1}^{(\ell)}=T_{m 0}^{\ell}(\varphi, \theta, \alpha) \sum_{s} \alpha_{s}^{(\ell)} \int f_{s}\left(r, v_{r}, v_{\perp}\right) v_{\perp} d v_{\perp} d v_{r}
$$

where we have defined

$$
\alpha_{s}^{(\ell)} \equiv\left|P_{\ell}^{s}(0)\right|^{2} .
$$

In order to derive, e.g., the dispersion relation (in the self-consistent problem) we need only to solve the Poisson equation. This gives the potential that corresponds to the density $\rho_{1}$. A comparison with the initial expression will then give the dispersion relation.

I shall illustrate this reduction procedure with a few examples.

\section{SPHERES WITH RADIAL ORBITS}

Let us derive, with the help of the reduction procedure, the equations describing the Jeans instability of a spherical system with radial orbits only. In this case, it is possible to derive a simpler set of equations. Since the equilibrium distribution function has the form

$$
f_{0}=\delta\left(L^{2}\right) \phi_{0}(E)=\frac{1}{r^{2}} \delta\left(v_{\perp}^{2}\right) \phi_{0}(E),
$$

i.e., contains a delta-function ( $E$ is the energy of radial motion, $E=v_{r}^{2} / 2+\Phi_{0}(r)$ ), the perturbation of the distribution function may be written ${ }^{2}$

$$
f_{s}=a_{s} \delta\left(v_{\perp}^{2}\right)+b_{s} v_{\perp} \delta^{\prime}\left(v_{\perp}^{2}\right)
$$

where $\delta^{\prime}$ is the derivative of the $\delta$-function, and the $a_{s}$ and $b_{s}$ functions depend only on $r$ and $v_{r}$ (and, of course, on the time $\left.t\right): a_{s}=a_{s}\left(r, v_{r}, t\right), b_{s}=b_{s}\left(r, v_{r}, t\right)$. For these functions we obtain the set of equations:

$$
\frac{\partial a}{\partial t}+v_{r} \frac{\partial a}{\partial r}+\frac{2 v_{r}}{r} a-\frac{\partial \Phi_{0}}{\partial r} \frac{\partial a}{\partial v_{r}}-\frac{1}{r} \frac{\partial b}{\partial \varphi}=\frac{1}{r^{2}} \frac{\partial \Phi_{s}}{\partial r} \frac{\partial \phi_{0}}{\partial v_{r}}
$$

2 An analogous procedure of $\delta$-expansions was first applied by Michailovskii, Fridman and Epel'baum (1970). 


$$
\frac{\partial b}{\partial t}+v_{r} \frac{\partial b}{\partial r}+\frac{3 v_{r}}{r} b-\frac{\partial \Phi_{0}}{\partial r} \frac{\partial b}{\partial v_{r}}=\frac{2 i s \Phi_{s}}{r^{3}} \phi_{0} .
$$

The density perturbation is

$$
\rho_{1}=4 \pi \sum_{s} \alpha_{s}^{(\ell)} \int d v_{r} a_{s}
$$

If we substitute $b_{s}=2 i s F_{1}$ and take into account that

$$
\sum_{s} \alpha_{s}^{(\ell)}=1, \quad \sum_{s} \alpha_{s}^{(\ell)} s^{2}=\frac{1}{2} \ell(\ell+1),
$$

then we obtain the following set of equations for the functions $F_{1}, a_{1} \equiv \sum_{s} \alpha_{s}^{\ell} a_{s}$ and $\chi(r)\left[\right.$ with $\Phi_{s}=\chi(r) e^{i s \varphi}$ and $\left.\hat{D} \equiv v_{r} \partial / \partial r-\left(\partial \Phi_{0} / \partial r\right) \partial / \partial v_{r}\right]:$

$$
\begin{gathered}
\frac{\partial a_{1}}{\partial t}+\hat{D} a_{1}+\frac{2 v_{r}}{r} a_{1}+\frac{\ell(\ell+1)}{r} F_{1}=\frac{\partial \chi}{\partial r} \frac{1}{r^{2}} \frac{\partial \phi_{0}}{\partial v_{r}} \\
\frac{\partial F_{1}}{\partial t}+\hat{D} F_{1}+\frac{3 v_{r}}{r} F_{1}=\frac{\chi}{r^{2}} \phi_{0} \\
\frac{1}{r^{2}} \frac{d}{d r}\left(r^{2} \frac{d \chi}{d r}\right)-\frac{\ell(\ell+1)}{r^{2}} \chi=4 \pi G \int a_{1} d v_{r},
\end{gathered}
$$

coinciding with the set in FP (derived in another way).

Further standard transformation of the set (18)-(20) consists of the natural substitution $a_{1} r^{2}=a$ and $F_{1} r^{3}=F$ in the expansions

$$
\begin{aligned}
a & =a_{+} \delta\left(v_{r}-v_{0}\right)+a_{-} \delta\left(v_{r}+v_{0}\right)+b_{+} \delta^{\prime}\left(v_{r}-v_{0}\right)+b_{-} \delta^{\prime}\left(v_{r}+v_{0}\right) \\
F & =F_{+} \delta\left(v_{r}-v_{0}\right)+F_{-} \delta\left(v_{r}+v_{0}\right)
\end{aligned}
$$

where $v_{0} \equiv \sqrt{2 E_{0}-2 \Phi_{0}(r)}$ and $\phi_{0}(E)=\int \phi_{0}\left(E_{0}\right) \delta\left(E-E_{0}\right) d E_{0}$. This gives

$$
\begin{gathered}
\frac{\partial a_{ \pm}}{\partial t} \pm v_{0} \frac{\partial a_{ \pm}}{\partial r} \pm v_{0}^{\prime} a_{ \pm}-\frac{\partial b_{ \pm}}{\partial r}+\frac{\ell(\ell+1)}{r^{2}} F_{ \pm}=0 \\
\frac{\partial b_{ \pm}}{\partial t} \pm v_{0} \frac{\partial b_{ \pm}}{\partial r} \pm 2 v_{0}^{\prime} b_{ \pm}=\frac{1}{2 v_{0}} \frac{\partial \chi}{\partial r} \\
\frac{\partial F_{ \pm}}{\partial t} \pm v_{0} \frac{\partial F_{ \pm}}{\partial r} \pm v_{0}^{\prime} F_{ \pm}=\frac{1}{2 v_{0}} \chi
\end{gathered}
$$

In particular, for the WKBJ-perturbations with $\ell \gg r(\partial / \partial r)$ the following symmetric set of equations can be obtained from (22)-(24) (Antonov, 1973):

$$
\hat{D}_{ \pm} \xi_{ \pm}=\frac{\zeta_{ \pm} \pm v_{0} \xi_{ \pm}}{r}
$$




$$
\hat{D}_{+} \zeta_{+}=\hat{D}_{-\zeta_{-}}=2 \pi G r \int d E_{0} \rho_{E_{0}}\left(\xi_{+}+\xi_{-}\right),
$$

where $\hat{D}_{ \pm}=(\partial / \partial t) \pm v_{0}(\partial / \partial r)=(d / d t)_{ \pm}$are the operators of the derivative over time along the unperturbed radial orbits of the particle "flow" with energy $E_{0}, \rho_{E_{o}}=\phi_{0}\left(E_{0}\right) / v_{0} r^{2}, \zeta_{ \pm}=-v_{0} F_{ \pm}$and $\xi_{ \pm}=\frac{1}{\ell^{2}} r v_{0} a_{ \pm}$. For perturbations of the form $\phi \sim Y_{\ell}^{m}(\theta, \varphi) \chi(r)$ the quantities $\xi_{ \pm}$and $\zeta_{ \pm}$may be thought of as the corresponding linear displacements in the equatorial plane in the azimuth $\varphi$ and the angular momentum $\left(r v_{\varphi}\right)$ of the particle. Proceeding from the system (25)(26), instability is proved by constructing the Lyapunov functional (Antonov, 1973; in English see FP).

The principal point in the derivation of the set of equations (25)-(26) was the use of the WKBJ-approximation in the angular variables [the condition $\ell \gg$ $r(\partial / \partial r)]$. Such an approximation would be justified if the eigenfunctions of such a type exist. Strictly speaking this point requires an additional justification. One may recall, for example, that eigenfunctions of such a kind are absent in the case of the exactly solved problem of perturbations of uniformly rotating disks with arbitrary elliptical orbits. On the qualitative level, the basis for the existence of such solutions in the case of systems with radial orbits requires a repetition of the arguments similar to those given above under the qualitative description of the instability mechanism. ${ }^{3}$

Proofs of the instability based on the application of equations (25)-(26) describing the short-wavelength perturbations say nothing about how systems with nearly radial orbits evolve in reality. For instance, the answer to the question of whether the initial spherical shape of the system changes depends on the stability or instability of just the largest scale modes. The answer to this question was obtained in the paper of the author mentioned above (Polyachenko 1981). It was shown that an initially spherical system with radial orbits converts in its non-linear evolution into an ellipsoidal one. At the same time in that paper (and also in those of our works where the boundary between stable and unstable systems was found) the radial-orbit instability itself was finally proved (without any approximations).

The problem of finding the eigenmodes $\left(\sim e^{-i \omega t}\right)$ in analytic form is in the general case rather complicated, even for the simplified set (25)-(26). However, in the particular case (important from the point of view of astronomical applications) when the system of particles is in a given external field (created, for example, by a more massive central condensation or by the "halo" which itself is not subjected to perturbations of such a type), ${ }^{4}$ we can advance rather far in the way of an analytical solution. The point is that in this case the first approximation is evidently the perturbation of the radial-orbit system in the given external potential, which can easily be determined. The gravitational interactions of particles with each other (their self-gravitation which causes the instability) may be accounted for in the next approximation. Thus, there appears the possibility of a good perturbation theory

3 Possibly all the systems may be separated into two classes: 1) those with nearly circular orbits (and "joining" to them) and 2) systems "joining" to those with radial orbits. For systems of the first class, the WKBJ-eigenfunctions with $\ell \ll r \partial / \partial r$ occur, and for systems of the second class, the eigenfunctions with $\ell \gg r \partial / \partial r$.

4 For example, the role of a "halo" may be played by a massive spherical system with an isotropic velocity distribution function of stars. 
in the small ratio $M / M_{h} \ll 1$ where $M_{h}$ is the "halo" mass, and $M$ is the mass of the radial-orbit system. Let us consider perturbations for which particles from one radial orbit (more exactly, a certain "flow" of particles with a fixed energy $E$ ) pass onto a nearby identical radial orbit. It is obvious that without taking into account the self-gravitation such perturbations correspond to a certain new equilibrium, i.e., in this approximation their frequency $\omega=0$. But the consistency condition for the solutions of the first and second (with self-gravity) approximations of the set (25)-(26) leads to the integral equation

$$
\omega^{2} F(E)=-4 \pi G \int_{0}^{r_{E}} \frac{d r}{v_{0}(r, E)} r^{2} \int d E_{0} \rho_{E_{0}}(r) \frac{F\left(E_{0}\right)}{F_{1}\left(E_{0}\right)}
$$

where

$$
F_{1} \equiv \int_{0}^{r_{E}} r^{2} \frac{d r}{v_{0}(r, E)}, \quad F(E)=\omega^{2} F_{1}(E) \xi_{E}
$$

In this case, the displacement $\xi(r, E)$ is proportional to the radius: $\xi=r \xi_{E}$, with $r_{E}$ the maximal radius for a particle with the energy $E: \Phi_{0}\left(r_{E}\right)=E$. Thus, $F(E)$ (or $\xi_{E}$ ) must be a solution of the integral equation (27), and the square of the eigenfrequency, $\omega^{2}$, is the eigenvalue of the corresponding integral operator.

Equation (27) can be easily transformed to the standard form of an integral equation with a symmetric kernel

$$
-\omega^{2} \bar{F}(E)=\int_{\Phi_{0}(0)}^{E_{\max }} \bar{K}\left(E, E_{0}\right) \bar{F}\left(E_{0}\right)
$$

where we introduced the new function

$$
\bar{F}(E)=F(E) \sqrt{f(E)}, \quad\left(f(E) \equiv \frac{2 \pi G \Phi_{0}(E)}{F_{1}(E)}\right),
$$

and the symmetric kernel is

$$
\bar{K}\left(E, E_{0}\right)=\sqrt{f(E) f\left(E_{0}\right)} \begin{cases}\int_{0}^{r_{E_{0}}} \frac{d r}{\sqrt{E-\Phi_{0}(r)} \sqrt{E_{0}-\Phi_{0}(r)}}, & E_{0}<E, \\ \int_{0}^{r_{E}} \frac{d r}{\sqrt{E-\Phi_{0}(r)} \sqrt{E_{0}-\Phi_{0}(r)}}, & E_{0}>E .\end{cases}
$$

In particular, it follows from (28)-(30) that for sign-definite eigenfunctions (for instance, $\bar{F}(E) \geq 0$ everywhere), $\omega^{2}<0$ and $\omega^{2} \simeq-G M / R^{3}$, i.e., instability with a growth rate of such an order takes place. For the simplest case of a homogeneous halo, $\bar{F}=\sqrt{4 G} \sqrt{\left.\phi_{0}(E) / E\right)} F(E)$ and

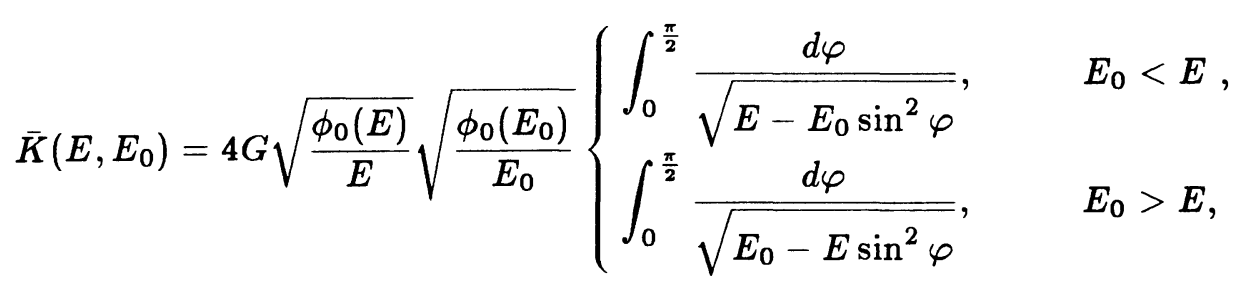


where the potential of a halo was taken as $\Phi_{h}=r^{2} / 2$. The integral equation (30) with kernel (31) was solved numerically for systems with various distribution functions $\phi_{o}(E)$. For instance, for $\phi_{o}(E) \propto E\left(E_{\max }-E\right)^{2}$ the growth rate of the most unstable mode (which corresponds to sign-constant $\xi_{E}$ with the maximum at $E=0)$ proved to be $\gamma \simeq 2.05 \sqrt{G M / R^{3}}$.

The integral equation (28) can be solved easily numerically for an arbitrary potential of a "halo" $\Phi_{k}(r)$; when the external field is that of a point mass, the integral equation (28) reduces to the following form (assuming $G M=1$ ):

$$
-\omega^{2} \bar{\chi}(x)=\int_{y_{0}}^{\infty} \bar{K}(x, y) \bar{\chi}(y) d y,
$$

where

$$
\bar{K}(x, y)=\frac{8 \pi G}{C x^{2} y^{2}} \sqrt{\phi_{0}(x)} x^{9 / 2} \sqrt{\phi_{0}(y)} y^{9 / 2} \begin{cases}\frac{x^{2}+y^{2}}{2 x y} \ln \frac{x+y}{y-x}-1, & y>x, \\ \frac{x^{2}+y^{2}}{2 x y} \ln \frac{x+y}{x-y}-1, & x>y,\end{cases}
$$

and

$$
\bar{\chi}(x)=\sqrt{\frac{8 \pi G}{C}} \sqrt{\phi_{0}(x)} x^{9 / 2} \chi(x)
$$

with

$$
C=\int_{-\infty}^{\infty} \frac{t^{6} d t}{\left(1+t^{2}\right)^{4}}=\frac{15 \pi}{16}
$$

\section{SPHERICAL SYSTEMS WITH A FINITE DISPERSION OF TRANSVERSE VELOCITIES}

Stability criteria for spherical stellar systems with general distribution functions were considered first in the papers by the author and Shukhman (1981; the main results are described in detail in FP). In these works the supposition was made that the stability (or instability) of systems with radially elongated orbits is determined by the value of the parameter of "global anisotropy" $\xi=2 T_{r} / T_{\perp}$ where $T_{r}$ and $T_{\perp}$ are the total kinetic energies corresponding to the radial and transversal degrees of freedom. For several series of distribution functions which differed greatly from each other, critical values of $\xi=\xi_{c}$ proved to lie in a rather narrow interval: between 1.4 and 2.0. The most interesting result is the fact that the anisotropy necessary for instability need not be too large: already for $\xi \gtrsim 2$ the system is usually unstable. (By the way, one sometimes considers models with a mean anisotropy $\xi \sim 5-8$, for instance, in some models of the stellar surroundings of massive black holes).

However, it is obvious that for a system immersed in a "halo" or containing a massive central cluster, i.e., in essence, compound systems, the criterion needs some improvement: this requires introducing the energy of interaction between an inner cluster (or a "halo") and surrounding stars in which the instability may develop. 
It is analogous to the necessity of accounting for the energy of interaction between disk stars and a "halo" in the Ostriker-Peebles criterion. Taking into account the Jeans nature of the instability, one may postulate a generalized instability criterion of the form:

$$
T_{r}>\xi\left(T_{\perp} / 2\right)+\eta U
$$

where $U$ is the modulus of the interaction energy between the stars in a "corona" and a central condensation or a halo (a more accurate definition of which is given below), $\xi$ is the critical anisotropy determined earlier, and $\eta$ is a number of order unity. Inequality (33) is, in essence, the condition for the transverse Jeans instability

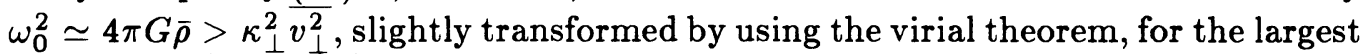
scale mode $(\kappa \sim 1\rangle R)$, which may be written in energetic terms as

$$
T_{\perp}<\alpha\left|U_{1}\right|
$$

where $U_{1}$ is the gravitational energy of the system under consideration ("corona"), and $\alpha$ is some constant. The gravitational interaction between the "corona" and the external gravitational field having been accounted for, the virial theorem gives

$$
2\left(T_{r}+T_{\perp}\right)=\left|U_{1}\right|+\int \rho\left(r \frac{d \Phi_{0}}{d r}\right) d V
$$

where $\rho$ is the mass density of the system, $\Phi_{0}$ is the external potential, and $d V=$ $4 \pi r^{2} d r . \quad U$ in the inequality (33) is, strictly speaking, simply the magnitude of the second term on the right hand side of the last formula (the "external" part of the virial): $U=\int \rho r\left(d \Phi_{0} / d r\right) d V$. Particularly, for a homogeneous "halo", writing $\Phi_{0}=\Omega_{h}^{2} r^{2} / 2$ (with $\Omega_{h}^{2}=$ const.) we have $U=2 \int \rho \Phi_{0} d V$, and for a point mass in the center, when $\Phi_{0}=-G M / r, U=\int \rho\left|\Phi_{0}\right| d V$. Expressing $\left|U_{1}\right|$ in equation (35) in terms of $T_{r}, T_{\perp}$ and $U$, we obtain

$$
2 T_{r}>\left(\frac{1}{\alpha}-2\right) T_{\perp}+U,
$$

i.e., the inequality (33), with $\xi=(1 / \alpha)-2$, and $\eta=1$.

It should be kept in mind that the simplest criteria for compound systems, such as inequality (33), operate with the kinetic energies $T_{r}$ and $T_{\perp}$ of the "corona" stars only, i.e., by our definition, of the component of a whole system that is responsible for the instability: only this component responds effectively to the most important perturbations (the fundamental mode, first of all-see footnote 3 ). If a finite - not necessarily massive - subsystem with nearly radial orbits may be distinguished in the whole system, it may be unstable, while the mean anisitropy of the whole compound system may be negligible (e.g., the ratio $2 T_{r}^{t o t} / T_{\perp}^{t o t}$ for the total system happens to be arbitrarily close to unity). This follows clearly from physical arguments, and, formally, from the inequality (34). In this case, the growth rate must be small, being of order of the Jeans frequency $\omega_{0}$ determined by the "corona" matter density.

We always have adopted just this interpretation of the instability conditions. This may be seen, for instance, in our critical comments on the paper by Duncan \& Wheeler (1980), who examined models of spherical systems with large anisotropy (see FP, Vol. 2, p. 151-153). Given a sufficiently small mass for the central anisotropic part of the system, the anisotropy of the whole system may be very small, $2 T_{r}^{\text {tot }} / T_{\perp}^{\text {tot }} \sim 1$. Nevertheless, perturbations localized near the centre (with a radial scale of order the size $\Delta r$ of the anisotropic region) must be unstable. ${ }^{5}$

5 This is valid with some reservations--see FP. 
This conclusion has recently been confirmed by Merritt and Aguilar (1985).

Thanks to Dr. Merritt, the paper by Palmer and Papaloizou presented at this Symposium became available to me. In essence, their result, which seems plausible, is that small angular momentum stars can form a distinct unstable subsystem-in the sense discussed above- if the distribution function $f_{0}\left(E, L^{2}\right)$ of the whole system becomes unbounded as $L \rightarrow 0$, for all $E$. However, the numerical results for the generalized polytropes given in this paper become invalid for the most interestingfundamental-mode, as they lie far beyond the framework of the approximations used. The stability of these models was studied earlier by us (Polyachenko 1983; in more detail, see FP): we showed that a pronounced instability, which develops in a few crossing times and leads to an easily perceptible ellipsoidal deformation of the whole system (and not only a small portion of it), occurs for anisotropies $2 T_{r} / T_{\perp} \gtrsim 1.4$. This result was obtained both by solving the corresponding matrix eigenvalue problem, and by $\mathrm{N}$-body simulations. The latter have recently been repeated by Barnes, Goodman and Hut (1986), using a different technique, and showing the same result.

It should be remarked that the last paper-as well as the paper by Palmer and Papaloizou - contains some critical comments resulting from the same misunderstanding, discussed above. These criticisms arise from a somewhat too blunt application of the instability criterion for the parameter $\xi=2 T_{r}^{\text {tot }} / T_{\perp}^{\text {tot }}$, which is assumed to be absolutely rigorous. Of course, this criterion is not valid in some cases, which we have already seen from general, reasonable considerations. One should realize that even physically more relevant criteria, like (33), may have only a preliminary, and very approximate character. They are useful, in our opinion, as a first step. Rigorous criteria are still absent. It is clear, however, that, when present, such criteria will be very complex, so that their applicability to the general case will still be questionable. Our criteria are simple, and cannot be "strongly" incorrect, since they are based on a simple and fairly obvious physical picture of the instability.

A way for isolating an "unstable subsystem" may be evident in some cases from the structure of the system considered. In other cases, this can be done only with some degree of uncertainty. However, for systems with a sufficiently smooth phase-space structure, one may apply the instability criterion $(\xi \gtrsim 2)$ directly for the value $\xi=2 T_{r}^{\text {tot }} / T_{\perp}^{\text {tot }}$ without any substantial error.

Concerning real star clusters and galaxies, they often have a strongly inhomogeneous structure, with a high concentration of matter in the center. The velocity distribution of stars in the central region is usually assumed to be isotropic. Such an isotropic region plays-roughly speaking - the role of a central mass which (cf. equation [33]) exerts a stabilizing influence on perturbations in an anisotropic "corona". 6

6 One may consider an isotropic (or any other) subsystem as an immovable halo when the stellar density response of this subsystem is negligible in comparison with that of an "unstable subsystem" of stars with elongated orbits. It is easy to write down the corresponding condition suitable for rough estimates. Conceptually, this problem is quite similar to that of the derivation of the condition under which it is possible to neglect perturbations in the spherical and other non-flat components of spiral galaxies when studying waves in the disk component (see, e.g., Marochnik $\&$ Suchkov 1974). By analogy, here we may write down a similar condition in the form: $\left(\rho^{(h)} / \rho^{(c)}\right) \cdot\left(c_{\perp}^{2(c)} / c_{\perp}^{2(h)}\right) \ll 1$, where $\rho^{(h)}$ and $\rho^{(c)}$ are the densities of a halo 
It is natural in such a situation to expect different critical values of anisotropy than for most of the models studied by us earlier. The simplest and rather realistic density distribution gives the well-known Plummer-Schuster law $\rho(r)=$ $\rho(0) /\left(1+r^{2} / r_{0}^{2}\right)^{5 / 2}$. The corresponding phase models were suggested by Kuzmin and Veltmann (1968). Their stability was investigated by us earlier, but the results presented, for example, in FP, had a rather large dispersion. The value $\xi_{c} \sim 1.6$ for three series of these models was obtained by means of a too-great extrapolation beyond the "large" growth rate values which were determined with confidence. At the same time, a smaller extrapolation, but taking into account rather small growth rates determined with less confidence, had led to values of $\xi_{c}$ closer to $\xi_{c} \sim 2$ (however, with a large dispersion). The restriction on $\xi_{c}$ from above in the form $\xi_{c} \lesssim 1.95$ arose just as a consequence of the latter determinations. Here we present the results defined more precisely (to be published in more detail elsewhere). It turned out that all the $\xi_{c}$ values lie within the narrow interval between $\sim 2.05$ and 2.10 for all physically acceptable models by Kuzmin-Veltmann (with phase densities positive everywhere).

\section{SPHERICAL SYSTEMS WITH NEARLY CIRCULAR ORBITS}

In the above we considered the systems with radially elongated orbits. The opposite type of anisotropy is that of clusters with nearly circular orbits. For such systems, one can obtain the dispersion relation describing short wavelength perturbations (like the well-known dispersion relation by Lin-Shu for disk galaxies):

$$
u x=1-\sum_{k} \alpha_{k}^{\ell} \frac{\nu_{k} \pi}{\sin \nu_{k} \pi} F_{x}\left(\nu_{k}\right)
$$

where

$$
\alpha_{k}^{\ell}=\left|P_{k}^{\ell}(0)\right|^{2}=(\ell+k) !(\ell-k) ! /\left[\left(\frac{\ell+k}{2}\right) !\left(\frac{\ell-k}{2}\right) ! 2^{\ell}\right]^{2},
$$

and $P_{k}^{\ell}$ are the associated Legendre functions, $\nu_{k}=\nu-\mu k / 2, \nu=\omega / \kappa, \omega$ is the eigenfrequency, $\kappa$ the epicyclic frequency, $\mu=2 \Omega / \kappa, \Omega$ is the angular velocity of the circular orbit, $\rho_{0}$ is the density, $u=\kappa^{2} / 4 \pi G \rho_{0}=4 /\left(4-\mu^{2}\right)$ for the self-consistent equilibrium,

$$
F_{x}(\nu)=\frac{1}{2 \pi} \int_{-\pi}^{\pi} e^{-x(1+\cos s)} \cos \nu s d s,
$$

$x=k^{2} c^{2} / \kappa^{2}, c$ is the radial velocity dispersion, and $k$ is the radial wavelength number. In the derivation of equation (37) the distribution function over $v_{r}$ was assumed to be Maxwellian. Branches of oscillations described by equation (37) for various $\ell$ resemble very much the cyclotron branches of plasma oscillations. There is a separate set of branches for each pair of $(\ell, \mu)$.

and a "corona", and $c_{\perp}^{(h)}, c_{\perp}^{(c)}$ are the transverse velocity dispersions. One might expect that this condition would be weakened if the "halo" occupied only a small central region. 
Considering systems with small values of $x$ and $\mu \simeq 4 / 3$ for $\ell=3$ one can find the small root: $\nu^{2} \simeq c x(\mu-4 / 3)$, with the constant $c$ equal to $\frac{25}{192}$, which implies instability if $\mu<4 / 3$. Analogous resonant conditions are fulfilled also for other values of $\ell$. Moreover, generally speaking, layers with arbitrary $\mu$ are unstable for one or another $\ell$. Thus, there exist mechanisms for isotropisation not only in systems with an increasing function $\Omega(r)$ when $\mu<1$ (this was known earlier) but also in normal systems with decreasing $\Omega(r)$.

\section{REFERENCES}

Antonov, V.A., 1960. Astr. Zh., 37, 918 [1961. Soviet Astronomy, 4, 859]. Antonov, V.A., 1962. Vestn. Leningrad Univ., 19, 96.

Antonov, V.A., 1973. in Dynamics of Galaxies and Star Clusters, p. 139, ed. Omarov, T.B., Nauka (Alma-Ata) (in Russian).

Barnes, J., Goodman, J., \& Hut, P., 1986. Astrophys. J., 300, 112.

Duncan, M.J., \& Wheeler, J.C., 1980. Astrophys. J., 237, L27.

Fridman, A.M., \& Polyachenko, V.L. 1984. Physics of Gravitating Systems, Vols. 1 and 2, Springer Verlag (FP).

Fridman, A.M., \& Shukhman, I.G., 1972. Dokl. Akad. Nauk SSSR, 202, 67 [Sov. Phys. Dokl., 17, 44].

Kuzmin, G.G., \& Veltmann, Yu I.K. 1968. Publ. Tartu Astroph. Obs., 36, 470.

Lynden-Bell, D., 1962. Mon. Not. R. astr. Soc., 124, 279.

Lynden-Bell, D., \& Sanitt, N., 1969. Mon. Not. R. astr. Soc., 143, 167.

Marochnik, L.S., \& Suchkov, A.A., 1974. Usp. Fiz. Nauk, 112, 275 |Sov. Phys. Usp., 17, 85].

Merritt, D.R., \& Aguilar, L.A., 1985. Mon. Not. R. astr. Soc., 217, 787.

Michailovskii, A.B., Fridman, A.M., \& Epel'baum, Ya.G. 1970. Zh. Eksp. Teor. Fiz., 59, 1608 [1971. Sov. Phys. JETP 32, 878].

Polyachenko, V.L., 1972. Kandidatskaja dissertatsija, Leningrad.

Polyachenko, V.L., 1981. Pis'ma Astr. Zh., 7, 142 [Soviet Astr. Lett., 7, 79].

Polyachenko, V.L., 1985. Astron. Tsirkular, 1405, 1.

Polyachenko, V.L., \& Shukhman, I.G., 1972. Inst. Zemn. Magn. Ionosf. Rasprostr. Radiovoln Sibir. Otd. Akad. Nauk SSSR, 2-72, Irkutsk.

Polyachenko, V.L., \& Shukhman, I.G., 1981. Astr. Zh., 58, 933 [Soviet Astronomy, $25,533]$.

Shukhman, I.G., 1973. Astr. Zh., 50, 651 [Soviet Astronomy, 17, 415].

Synakh, V.S., Fridman, A.M., \& Shukhman, I.G., 1971. Dokl. Akad. Nauk SSSR, 201(4), 827 [1972. Sov. Phys. Dokl., 16(12), 1062].

Vilenkin, N.Ya. 1968. Special Functions and Group Theory, Nauka, Moscow (in Russian). 